\title{
Integrated learning of saliency, complex features, and object detectors from cluttered scenes
}

\author{
Dashan Gao \\ Nuno Vasconcelos \\ Department of Electrical and Computer Engineering, \\ University of California, San Diego \\ dgao@ucsd.edu \\ nuno@ece.ucsd.edu
}

\begin{abstract}
A novel procedure for object detection from cluttered scenes is proposed. It consists of an integrated solution to the problems of learning 1) a saliency detection module tuned to a class of objects of interest, 2) a set of complex features that achieves the optimal trade-off, in a minimum probability of error sense, between discrimination and generalization ability, and 3) a large-margin object detector. All stages of the new procedure have some degree of biological motivation and this is shown to enable a computationally efficient solution that is scalable to problems containing large numbers of object classes. Experimental evidence is given in support of the arguments that different levels of feature complexity are optimal for different object classes, and that optimal features range from parts to templates, depending on the variability of the object class.
\end{abstract}

\section{Introduction}

With the formulation of object detection and recognition as statistical classification problems and the advent of powerful classification architectures, the last decades have witnessed major improvements in detection and recognition accuracies. Yet, there are still various aspects in which the current state of understanding of these problems is too limited to allow the design of systems with the robustness and flexibility required by most practical applications. One of the significant limitations of current recognizers is a requirement for carefully controlled training, usually performed with large training sets that are manually assembled and pre-processed. This results in extremely lengthy data collection procedures that make it difficult to rapidly deploy a classifier for a given class of objects, if a training set is not already available for that class.

Lately, however, the vision community has started to investigate a new formulation of the detection/recognition problem, usually referred to as recognition from cluttered scenes, where it is assumed that the training examples are not previously segmented $[2,5,4,9,10]$. For example, a training set of faces will contain images where the faces are shown in front of some background scene that occupies the bulk of the image area. One aspect that makes the new formulation fundamentally different from the traditional, uncluttered, learning problem is the very unbalanced nature of the available example labels. While in the "negative" class every image region can be confidently assumed to be a "negative" example, for the "positive" class the situation is quite different. In fact, while each training image in this class is labeled as containing the object of interest, it is not clear which image regions are really "positive" or "negative" examples. This implies that every image neighborhood could potentially be of interest for learning, and leads to a potentially very large (and noisy) training set. Hence, in addition to the standard problems in detection and recognition (how to find good features, how many should be used, how to design an effective classifier) recognition from cluttered scenes requires the ability to learn which regions of each training image are informative for the task at hand, namely which regions contain the objects of interest.

This can be seen as a saliency problem, i.e. the problem of determining the image regions that are salient for detection/recognition purposes. Given a reasonable saliency module, it should be possible to extract a set of image regions containing the objects of interest, and then apply to this training set (complemented with a set of negative examples which are usually easy to find) any of the existing procedures for the design of object detectors or recognizers. Overall, the problem has two major components: 1) the identification of training examples and 2) the design of the classifier itself. Given that neither the saliency nor the classification stage are likely to be perfect, it appears that significant gains might be possible by integrating the two stages. The classifier should certainly improve when saliency is more accurate (because it will have access to a cleaner training set) and the saliency stage should be able to 
improve with feedback from the classifier (regarding image regions that it considered salient but were clearly identified by the classifier as not containing the object of interest).

This is the problem addressed by this work, where we present an integrated solution for saliency and classification in the context of object detection problems. The work includes various contributions that address significant open questions for this problem. The first is a discriminant formulation of saliency that is optimal in a classification sense and produces saliency locations which are most informative in the sense of identifying the object of interest. The second is an iterative procedure that relies on classification results to improve saliency, and on the improved saliency results to obtain a better classifier. The third is a procedure to generate a hierarchy of features of increasing complexity, which allows fine control over the trade-off between discriminant power (which increases with complexity) and generalization ability (which tends to decrease with increasing complexity). The fourth is a biologically inspired, and computationally efficient, mechanism for feature selection from overcomplete feature sets, that balances discrimination and redundancy reduction.

Overall, our results show that it is possible to simultaneously learn, in a strictly discriminant fashion, 1) a saliency detection module tuned to the object class of interest, 2) a set of complex features that achieve an optimal trade-off between discrimination and generalization for the detection of objects in that class, and 3) a large-margin object detector. It is also shown that different levels of feature complexity are optimal for different object classes, and that the optimal features range from parts to templates, depending on the variability of the class. All stages of our algorithm have some degree of biological motivation and this is shown to enable a computationally efficient solution that is scalable to problems containing large numbers of object classes, without compromising optimality in a classification sense.

\section{Related work}

Learning to segment and recognize objects from cluttered scenes is a topic that has received an increased amount of attention in recent years $[1,2,5,4,9,10]$. A common theme to current approaches to this problem is to represent an object as a collection of parts. This leads to two fundamental questions: how to extract these parts from cluttered images, and how to represent them. The first problem is usually solved in one of two ways. The first is to randomly crop image patches from the images in the training set, at a wide range of scales, and then select those which are informative with respect to the object class [4]. This is a strategy of least commitment which guarantees that no fundamentally important patches will be lost due to coarse sampling. On the other hand, in order to guarantee coverage

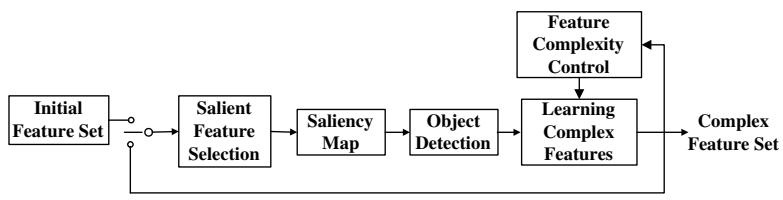

Figure 1. A hierarchical model for integrated learning of saliency maps, object detector, and complex features.

of the object of interest in its entirety, this approach usually requires sampling a very large number of image locations. This makes the subsequent step of patch selection computationally intensive and, so far, this method has only been demonstrated on databases of small images.

An alternative approach is to rely on a saliency detector to find a set of "interest points" in each training image $[2,5,9,10]$. While drastically more efficient, from a computational point of view, this approach has weaker performance guarantees from an accuracy standpoint, because the definitions of saliency in current used are unrelated to the detection problem. Instead, saliency is usually defined as some universal property that salient image regions must exhibit. Particularly popular definitions are that the image region must 1) contain specific visual attributes, such as edges or corners [6], or 2) exhibit a significant amount of complexity, where complexity can be defined in multiple ways $[8,7]$. Since these definitions do not constrain salient regions to be informative with regards the detection problem (e.g. are not tuned in any form to the class of objects to be detected) they tend to produce a collection of interest points that are only weakly guaranteed to have any relation to the object of interest. This increases the difficulty of the design of representative object parts, which has to be very robust to the presence of training outliers. The complexity is, in this way, shifted to the representation stage, which tends to be computationally intensive for these methods.

With respect to the representation of object parts, while some have argued for the use of simple features (e.g. local descriptors such as PCA, or SIFT [7]) [5, 9, 10], others have proposed complex ones (image patches) [2, 4]. Being more closely tuned to the objects of interest, complex features are certainly more discriminant. On the other hand, the response of simple features tends to exhibit less variability when images are subject to spatial image deformations, noise, or other perturbations. Overall, feature complexity is, for object detection, the main variable for controlling the trade-off between discriminant power and generalization ability (invariance) faced by any classifier. It therefore appears that best results should stem from 1) considering a hierarchy of features that span the continuum from simple to complex, and 2) learning the appropriate level of feature complexity for each detection problem. 


\section{Integrated saliency and object detection}

In this work, we address all problems discussed above by proposing an integrated solution for learning saliency maps, object detectors, and features. In particular we propose an iterative procedure, illustrated by Figure 1, consisting of the following steps. We start by selecting the most discriminant subset among a set of simple features (the discrete cosine transform - DCT - descriptors), which is used to generate a discriminant saliency map. Image patches are then extracted from the most salient locations and used to train an object detector. Using standard cross-validation it is possible to identify which patches are most likely to be positive examples, and these are passed to a feature extraction module. This consists of finding the best approximation to each of the salient patches, at a given level of spatial resolution. The resulting features are more complex than the initial set and more tuned to the object class of interest. The process is then iterated and the spatial resolution of the features (complexity) allowed to increase at each iteration. The result is a feature hierarchy, that ranges from simple (DCT descriptors) to complex (image patches), allowing explicit control of the trade-off between discriminant power and generalization ability. A saliency map and an object detector are also produced at each level of this hierarchy.

\subsection{Salient feature selection}

To avoid the lack of specificity of existing saliency detectors, we rely on a new form of saliency, which we refer to as discriminant saliency, and is intrinsically grounded on the recognition problem [11]. In particular, we equate the saliency of each region with how much it contributes to the solution of the detection problem, by defining the salient attributes of a given visual concept as the attributes that most distinguish it from all other visual concepts that may be of possible interest. Due to this equivalence between saliency and discrimination, discriminant saliency can be naturally formulated as an optimal feature selection problem: the most salient features are the ones that best separate the class of interest from all other classes.

\subsubsection{Scalable feature selection}

Since saliency is only a pre-processing stage to object detection, the process of salient feature selection should be computationally efficient. The design of optimal feature selection methods of low-complexity is a problem that we, and others, have been actively pursuing in the context of research in feature selection itself [11]. Our research has shown that information-theoretic methods, based on maximization of mutual information between features and class labels, have the appeal of enabling a precise control (through factorizations based on known statistical properties of images) over the trade off between optimality, in a minimum Bayes error sense, and computationally efficiency [11]. Our experience of applying algorithms in this family to the saliency detection problem is that, when the starting feature set is complete, even those strongly biased towards efficiency can consistently select good saliency detection filters. This is illustrated by all the results presented in this paper, where we have adopted the maximization of marginal diversity (MMD) [12] as the guiding principle for feature selection.

Given a classification problem with class labels $Y$, prior class probabilities $P_{Y}(i)$, a set of $n$ features, $\mathbf{X}=$ $\left(X_{1}, \ldots, X_{n}\right)$, and such that the probability density of $X_{k}$ given class $i$ is $P_{X_{k} \mid Y}(x \mid i)$, the marginal diversity (MD) of feature $X_{k}$ is

$$
\operatorname{md}\left(X_{k}\right)=<K L\left[P_{X_{k} \mid Y}(x \mid i) \| P_{X_{k}}(x)>_{Y}\right.
$$

where $<f(i)>_{Y}=\sum_{i=1}^{M} P_{Y}(i) f(i)$, and $K L[p \| q]=$ $\int p(s) \log \frac{p(x)}{q(x)} d x$ the Kullback-Leibler divergence between $\mathrm{p}$ and $\mathrm{q}$. Since it only requires marginal density estimates, the MD can be computed with histogram-based density estimates leading to an extremely efficient algorithm for feature selection. Furthermore, in the one-vs-all classification scenario, the histogram of the "all" class can be obtained by a weighted average of the class conditional histograms of the image classes that it contains, i.e.

$$
P_{X_{k} \mid Y}(x \mid \mathcal{A})=\sum_{i \in \mathcal{A}} P_{X_{k} \mid Y}(x \mid i) P_{Y}(i)
$$

where $\mathcal{A}$ is the set of image classes that compose the "all" class. This implies that the bulk of the computation, the density estimation step, only has to be performed once for the design of all saliency detectors. In summary, for complete feature dictionaries, the discriminant saliency procedure is highly scalable in the number of object detectors to learn.

\subsubsection{Saliency map and salient location}

Our experience is that, given a set of discriminant features, remarkably simple mechanisms, inspired by biological vision, are sufficient to achieve good saliency results. In particular, we have adopted a two step procedure based on the classical Malik-Perona model of texture perception [13], illustrated in Figure 2. First, a saliency map (i.e. a function describing the saliency at each image location) is obtained by pooling the responses of the different salient features after half-wave rectification

$$
S(x, y)=\sum_{i=1}^{2 n} \omega_{i} R_{i}^{2}(x, y),
$$




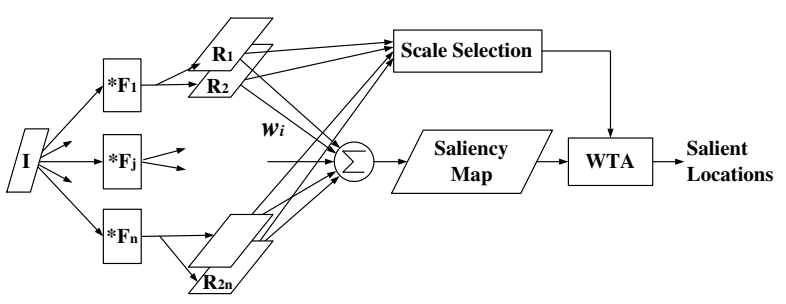

Figure 2. Schematic of the saliency detection model.

where $S(x, y)$ is the saliency at location $(x, y)$, $R_{i}(x, y), i=1, \ldots, 2 n$ the channels resulting from half-wave rectification of the outputs of the saliency filters $F_{i}(x, y), i=1, \ldots, n$

$$
\begin{array}{r}
R_{2 k-1}=\max \left[-I * F_{k}(x, y), 0\right] \\
R_{2 k}=\max \left[I * F_{k}(x, y), 0\right]
\end{array}
$$

$I(x, y)$ the input image, and $w_{i}=m d(i)$ a weight equal to the feature's marginal diversity. Second, the saliency map is fed to a peak detection module implemented as a winnertake-all network. The location of largest saliency is first found. Its spatial scale is set to the size of the region of support of the salient feature with strongest response at that location. All neighbors within a circle whose radius is this scale are then suppressed (set to zero). The process is iterated and produces a list of salient locations, their saliency strengths, and scales.

\subsubsection{Over-complete feature sets}

Unlike simple generic features, such as wavelet or DCT filters, class-specific features sets learned from training image collections tend to be highly over-complete. This implies that they contain subsets of features which are highly redundant. These redundancies must be penalized during feature selection, since the selection of a feature that is highly redundant with a previously selected one does not add much discriminant power (independently of how discriminant the new feature is on its own).

Feature selection in the presence of strong dependencies is, computationally, a much more demanding process than when such dependencies are not present. In particular, accounting for dependencies requires either 1) modeling joint densities, a process that has exponential complexity in the order of the dependency sets, or 2) penalizing the training samples that are well explained by the previously selected features, at each round of feature selection, as is done in methods such as boosting [14]. Our experience with various existing feature selection methods, from both camps, is that that they would significantly compromise the computational efficiency of discriminant saliency detection.

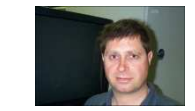

(a)
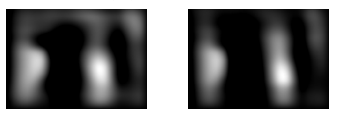

(c)

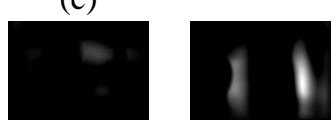

(d)

(b)

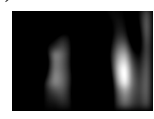

)
Figure 3. Illustration of non-maximum suppression. (a) A reference image. (b) Three features (c) Saliency maps (d) Saliency maps after suppressoin.

Furthermore, we would like to embed feature selection in the computation of the saliency map itself, as some dependencies may have great impact on the latter while others may be irrelevant. To achieve this goal, we propose a biologically inspired feature selection procedure that combines aspects of the two feature selection strategies. As in the case of the simple features, we start by ordering the feature set according to the MMD criterion. We then pick features sequentially, in a manner that maximizes discrimination but penalizes redundancies. This penalty is accomplished with recourse to the prime biological mechanism for redundancy reduction, non-maximum suppression, which results in an example-reweighting method for feature selection.

The use of non-maximum suppression to penalize dependencies is probably best understood with respect to Figure 3, which presents an example in the context of face detection. A set of features, shown in (b), is initially available in result of the MMD-based selection step. These features are highly discriminant (they look like faces or components of faces) but also redundant. Typical redundancies include similar features at slightly different scales, shifted replicas of the same feature, etc. A reference image, shown in (a), is first randomly selected and individual saliency maps produced for that image by considering one feature at a time. The individual saliency maps are shown in (c). The largest response among these saliency maps is then found, and the corresponding feature selected. Non-maximum suppression then consists of subtracting the saliency map of the selected feature to all the others. The process is iterated until either 1) all features are selected, or 2) all the remaining saliency maps are below a threshold (set to zero in all results presented in this paper). Our experience is that the latter invariably occurs much earlier than the former.

The example of Figure 3 contains three features, two that are highly redundant (full-face detectors of slightly different 
scale shown on the leftmost slots of (b)) and a third (a face part shown in the rightmost slot) that has small redundancy with them. In result, the saliency maps of the first two features are quite similar and significantly different from that of the third. Because the full-face features are most discriminant for face detection, the leftmost feature is selected in the first round. The middle feature is also highly discriminant, but due to its redundancy with the first, the subtraction of the two saliency maps is nearly zero. On the other hand, the saliency map of the rightmost feature is not significantly affected by the suppressium, and this feature becomes the winner in the second round, even though it is individually less discriminant that the one in the middle. Overall, the combined process of feature selection and generation of the saliency map has great computational efficiency.

\subsection{Learning complex features}

The saliency maps of the previous section can be seen as soft segmentation masks for the object of interest. In this section we present a method that relies on these masks to learn complex features tuned to that object. So far, we have not addressed the problem of how to determine the number of features that contribute to a given saliency map. In general, it is important to limit the number of such features, because there are usually many which are not discriminant. Non-discriminant features usually have a strong response in image regions that do not cover the object of interest, therefore degrading the saliency map. If the features are simple, even the saliency map originated by the best feature subset can have outliers, since the content of some image locations may be indistinguishable from the object of interest in the feature space under consideration.

Given that extracting complex features from areas not covered by the object of interest is likely not to be very useful, the extraction of complex features requires 1) determination of the best number of simple features to use in the construction of saliency maps, and 2) elimination of outlier locations in the resulting saliency maps. We refer to the combined process as the extraction of representative object locations. Once it is done, a collection of object patches can be obtained by retrieving the locations of largest saliency, and a new set of features, more complex and tuned to the object, can be learned. We refer to this process as the generation of complex features.

\subsubsection{Extraction of representative locations}

We adopt a cross-validation strategy for the extraction of representative locations. The basic idea is to start from the saliency maps associated with images that contain the object of interest and extract image patches located at the points whose saliency is above a threshold (some examples are shown as circles in Figure 4). This produces a set of positive (object) examples. Repeating the process on images known not to contain the object produces a set of difficult negative (non-object) examples. The two sets are then used to learn a classifier using standard cross-validation techniques. The process is repeated for all possible numbers of simple features used in saliency map design. By monitoring test error it is possible to determine the optimal value for that number.

The main source of difficulties for this procedure is that the training set of the positive class is, in general, corrupted. Because saliency is not perfect, there are usually outlying background patches which survive the saliency filter. To increase the robustness to this problem, we adopt as measure of classifier goodness the probability of error on the task of classifying training images, rather than patches. After all, this is the only data for which there is unmistakable ground truth. Images are classified in two steps. First, the image patches extracted from an image by the saliency detection stage are classified individually. Next, if at least one of the individual patches is classified as positive, the image is assigned to the object class. If all patches are negative, the image is assigned to the negative class. The ROC equal error rate (i.e. $p($ Truepositive $)=1-p($ Falsepositive $)$ of the resulting detector is used as a measure of the performance.

We believe that the exact classifier architecture used to classify the individual patches is not a determinant factor in the overall performance, as long as a good classifier is obtained. In our experiments we have used a support vector machine, due to its well known generalization ability and the availability of efficient implementations. The SVM learns a decision boundary of the form

$$
f(x)=\operatorname{sign}\left(\sum_{i} a_{i} y_{i} K\left(x_{i}, x\right)+b\right)
$$

where $K(x, y)$ is a kernel function that transform data to a high dimensional space, $b$ a bias term, and $x_{i}$ and $a_{i}$ are a set of support vectors and their weights. SVMs are best understood in the context of classifying separable data, i.e. when there is no overlap between the positive and negative classes. When this is the case, the SVM parameters (support vectors and weights) are learned so as to find the classification boundary with the maximum margin, where the margin is defined as the distance from the closest examples to the boundary. Support vectors are the examples closest to the boundary (i.e. at a distance from it equal to the margin). The problem is usually normalized so that margin is equal to 1 . Given a test example, the SVM computes the distance from the example to the boundary and thresholds it by the bias. The larger (and positive) this distance, the greater the confidence that the example is from the positive class.

For non-separable data, the SVM formulation is slightly 

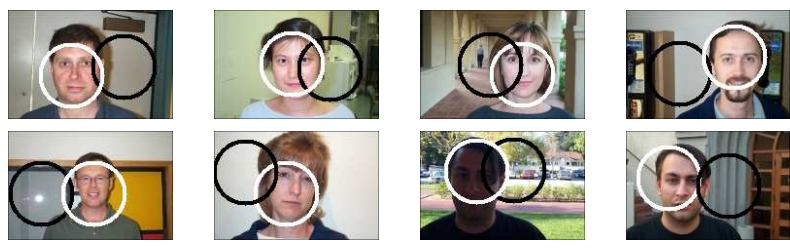

Figure 4. Examples of image patches accepted (white circles) and rejected (black circles) by the SVM.

more complex, we omit the details which can be found in various texts [15]. The most relevant point, in the context of this work, is that the confidence measure provided by the distance to the classification boundary generalizes to this case. It can therefore be used as a measure for selecting the image patches from the positive class to be passed to the next stage, where complex features are generated. We adopt the strategy of selecting the positive patches that have a distance to the boundary superior to the margin. Figure 4 shows a collection of images with saliency detection errors and the results of the SVM classification. White circles indicate patches accepted by the classifier, while black circles indicate their rejected counterparts.

\subsubsection{Generation of complex features}

The image patches extracted from representative locations are usually good prototypes for the object of interest. Nevertheless, because they are extracted from particular images, they tend to be too specifically tuned to the particular object and viewing conditions captured by those images. It is, therefore, unlikely that they will generalize well if directly used as features for object detection [3]. Instead, good features must balance discrimination with robustness to variations in object appearance, in order to guarantee good generalization. As suggested in [3], one possibility to increase robustness is to reduce spatial resolution (or complexity). For example, the variation in the response of a feature of high frequency content to a variation in object pose is likely to be larger than that of a low-pass filtered replica.

It follows from this argument that feature complexity is naturally equated with spatial resolution. To generate features with a given level of complexity, we therefore propose to approximate the salient image patches by the best linear combination of a pre-specified number of simple features. In particular, if $\mathbf{I}$ is a salient image patch and $\left\{b_{1}, b_{2}, \ldots, b_{N}\right\}$ a set of $N$ simple features, the best approximation of complexity $k$ is defined as the subset of $k$ features whose linear combination best approximates the patch in the least squares sense

$$
\min _{\substack{n_{1} \ldots n_{k} \\ a_{n_{1}} \ldots a_{n_{2}}}}\left\|\mathbf{I}-\sum_{i=1}^{k} a_{n_{i}} b_{n_{i}}\right\|^{2}, 1 \leq n_{i} \leq N
$$

If the set of simple features is orthogonal, the coefficients $a_{i}$ are obtained by projecting $\mathbf{I}$ onto the space spanned by the features $b_{i}$. The optimal set $b_{n_{i}}, i=1, \ldots, k$ is that containing the $k$ features of largest coefficient magnitude. When the simple feature set is complete, the approximation error is monotonically decreasing on $k$ and can always be made zero by making $k=N$. In this work we rely on a set of $8 \times 8$ DCT features to compose the simple feature set. This set is orthogonal and complete.

\section{Results and discussion}

The performance of the proposed object detection architecture was evaluated on the Caltech database, using the experimental set up proposed in [5].
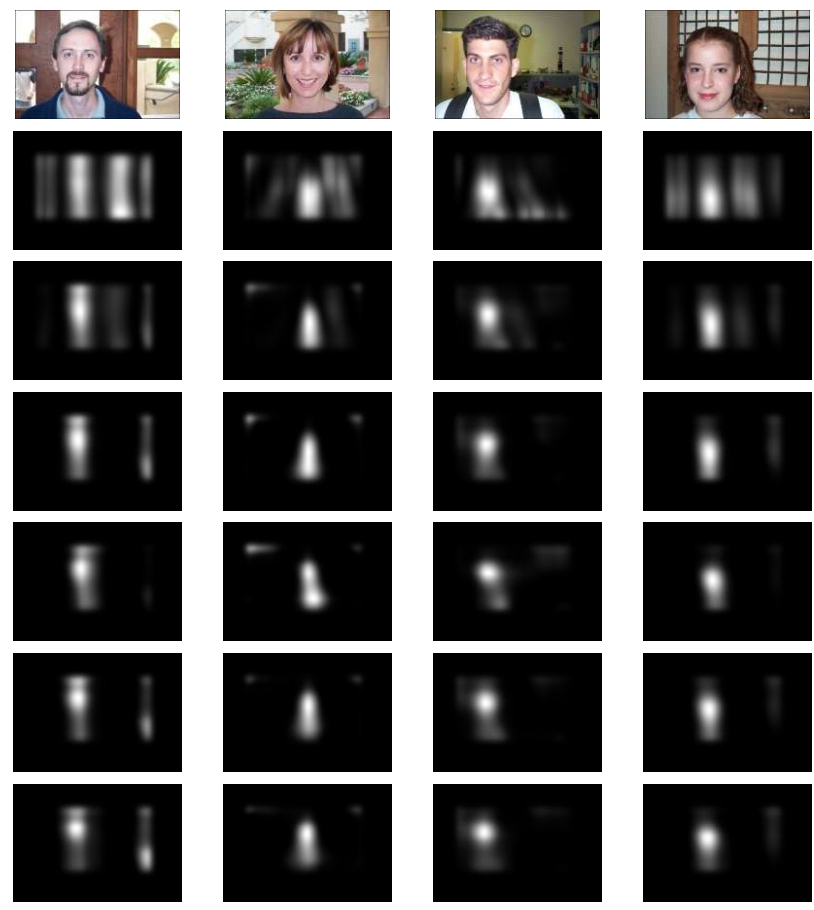

Figure 5. Saliency maps generated with features of different complexity. Saliency maps shown from the second to the last row were generated with 1, 4, 8, 16, 32 and 64 linearly combined DCT features. 


\subsection{Performance of complex salient features}

Figure 5 shows some examples of saliency maps generated at different stages of the feature complexity hierarchy for the face class. They were obtained with subsets of complexity $k \in\{1,4,8,16,32,64\}$ from the DCT set. The simple and generic features used in the first stage appear to be sufficient for some scenes, but are not very selective for others, where they respond quite strongly to various areas of background. It is, however, clear that even for the simplest features the face regions always originate a strong response. At later stages, where the saliency is computed with complex features, the response is clearly stronger on the faces areas than the background, for all scenes.
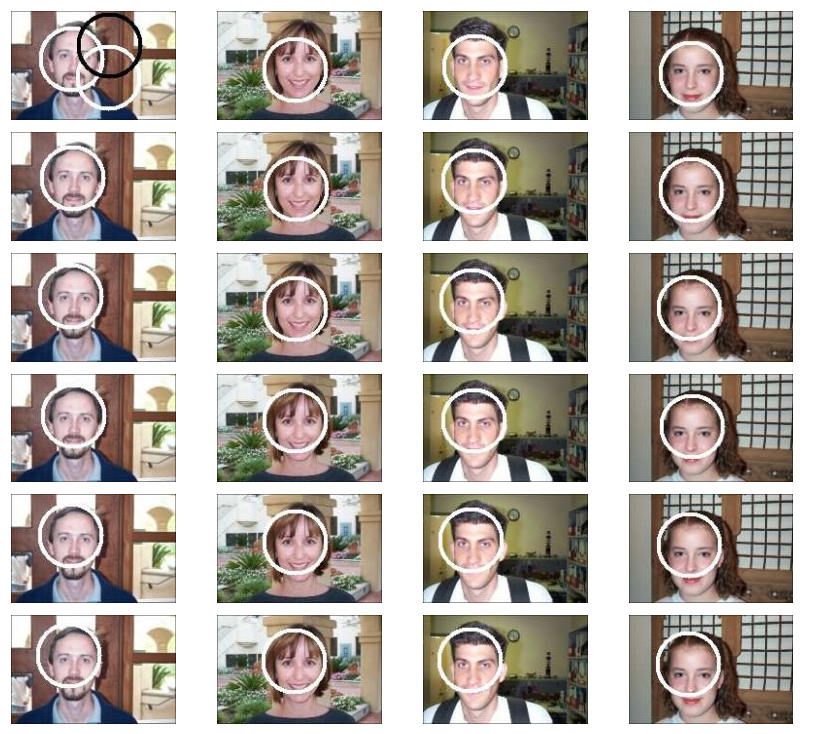

Figure 6. Salient locations detected with features of different complexity. From the second to the last row: $k \in$ $\{1,4,8,16,32,64\}$ respectively. White circles are salient locations accepted by the SVM classifier, black circles indicate the ones that are rejected.

To evaluate the saliency maps objectively we compared them, as well as the resulting detected salient locations (shown in Figure 6), with ground truth, manually obtained by placing a rectangle around each face. The results are shown in Figure 7. The first measure that we computed was the percentage of the total energy of the saliency map that was contained in the ground truth box. Figure 7(a) presents the cumulative sum of this measure for features of different complexity. It can be observed that the saliency is more spead over the image for simple features than for complex features, confirming the observations of Figure 5. However, in most cases, the bulk of the saliency energy is contained in the ground truth box. For example, the saliency maps generated by simple features have more than $70 \%$ of their energy inside the ground truth area $13 \%$ of the times. For more complex features this percentage is always above $50 \%$.

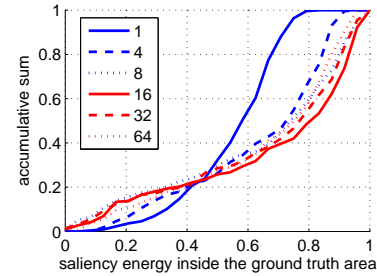

(a)

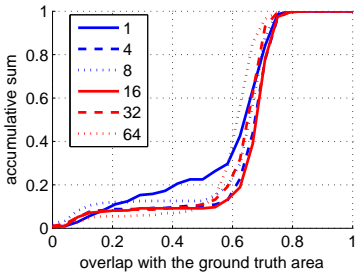

(b)
Figure 7. Comparison between saliency maps, generated with features of different complexity, and the ground truth on the face database. Cumulative distribution of (a) percentage of salient energy inside the ground truth box, and (b) overlap between salient locations and ground truth.

The second measure, whose cumulative sums are shown in Figure 7(b), is the relative overlap between the bounding box of the most salient location and the ground truth. If $A$ and $B$ are two bounding boxes, the relative overlap is defined as

$$
\operatorname{overlap}(A, B)=\frac{|A \cap B|}{|A \cup B|}
$$

where $|A|$ is the area of $A$. Again, complex features show better performance, but the differences are less significant. This indicates that, even though the simple features respond more strongly in non-face areas, the strongest response is already quite reliably aligned with the face location. Overall, the best results are obtained with complex features composed by a linear combination of 16 simple features.

This result is probably best understood by considering the salient features learned at each stage, which are shown in Figure 8. In the first stage salient features tend to be vertical bars, and contain only very low frequency information about face. As the complexity increases, and more high frequency information is added to the features, these start to look more like faces. In the final stages, where all 64 simple features are used to represent each complex feature, the features become face templates cropped from individual images. As discussed above, these templates are too tuned to individual faces and cannot account well for the variation inside the face class. In result, they lead to worse saliency maps than the ones of intermediate complexity.

We finalize the discussion on saliency by presenting, in Figure 10 and 11, saliency maps generated by features of complexity $k \in\{1,4,8,16,32\}$ for the airplane and motorbike classes. In general terms, the conclusions derived for the face class hold for these classes as well. The only significant difference is that, while for faces the learned complex features tend to be templates, for these classes they are 


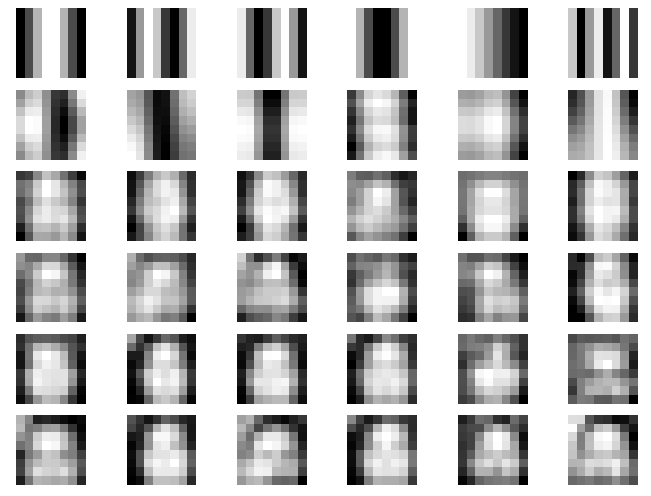

Figure 8. Six salient features generated at each stage of the feature complexity hierarchy. From top to bottom: complex features generated by combination of $1,4,8,16,32$ and 64 DCT features.

parts, as can be seen from Figure 9. This shows that optimal complex features can range between the two types, depending on the variability of the object class.

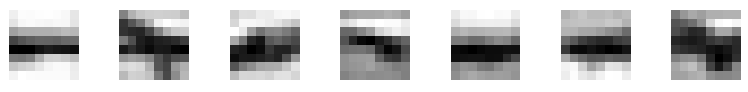

Figure 9. Seven of the salient features learned for Airplanes by combinations of 32 DCT features.

\subsection{Object detection}

In this section, we evaluate the performance of the SVM classifiers at each stage of the hierarchy. Figure 12(a) presents the ROC equal error rates obtained at the different stages. For the face class, and consistently with the results of Figure 7, the best performance is achieved with the complex features of complexity $k=16$. It is quite interesting, however, to realize that for the other two classes, motorbike and airplanes, simpler features actually work best. From the images of these two classes, shown in Figures 10 and 11, it is clear that these classes contain significantly more variability in appearance, pose, and scale than the faces. It is, therefore, not surprising that the performance of the complex features degrades in this case.

Another interesting observation is that, although there are mislabeled examples in the positive training set used to design the classifiers at all stages, these classifiers do not exhibit great difficulty in eliminating the mislabeled image patches and, consequently, generate good candidate features for the next stage. This is illustrated by Figure 4 . Figure 12(b) shows the percentage of examples accepted by the SVM at each stage. It can be seen that the classifiers

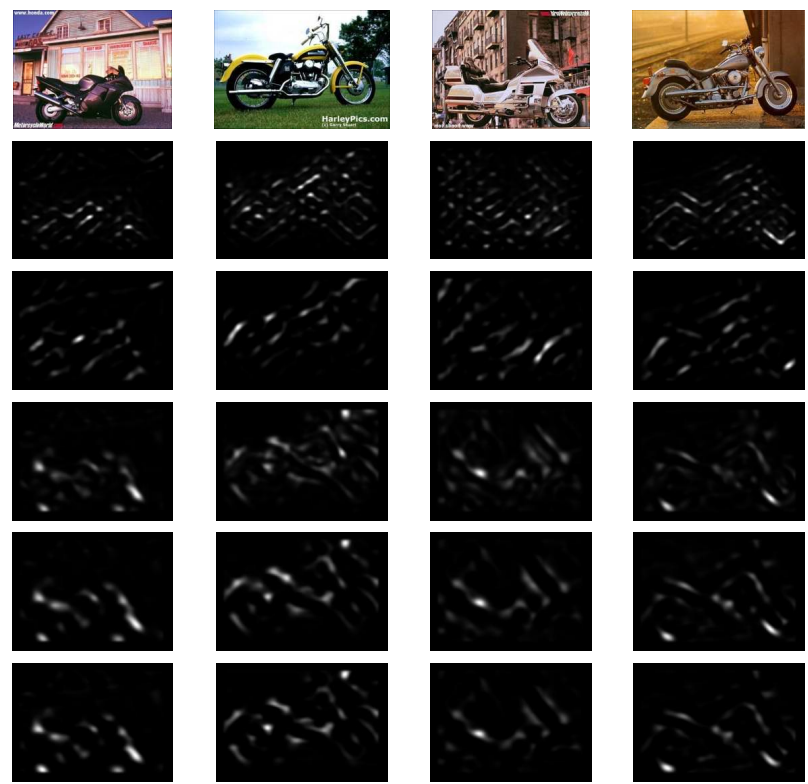

Figure 10. Saliency maps generated with features of different complexity, for the bike class. From the second to the last row: $k \in\{1,4,8,16,32\}$.

tend to reject more examples in the earlier stages, where the lower quality of the saliency maps leads to a higher number of mislabeled positive examples. At the later stages, where there are fewer saliency outliers, the classifier tends to accept more examples.

We would like to finish by pointing out that all results presented were obtained with a straightforward implementation of the algorithmic procedures discussed in the text. There are many operations that could be implemented to improve performance but which we have, so far, not considered. These include pre-processing operations, such as compensating for illumination variations by histogram equalization or subtraction of a dominant gradient, or resampling operations, such as augmenting training sets with perturbed replicas of the training examples (so as to obtain better invariance to various transformations). The benefits of including such operations remain an open subject for future research.

\section{References}

[1] Y. Amit and D. Geman, "A computational model for visual selection," Neural Computation, Vol.11(7), pp.1691-1715, 1999

[2] S. Agarwal and D. Roth, "Learning a sparse representation for objection detection," In Proc. ECCV 2002, Vol. 4, pp. 113-130, 2002. 

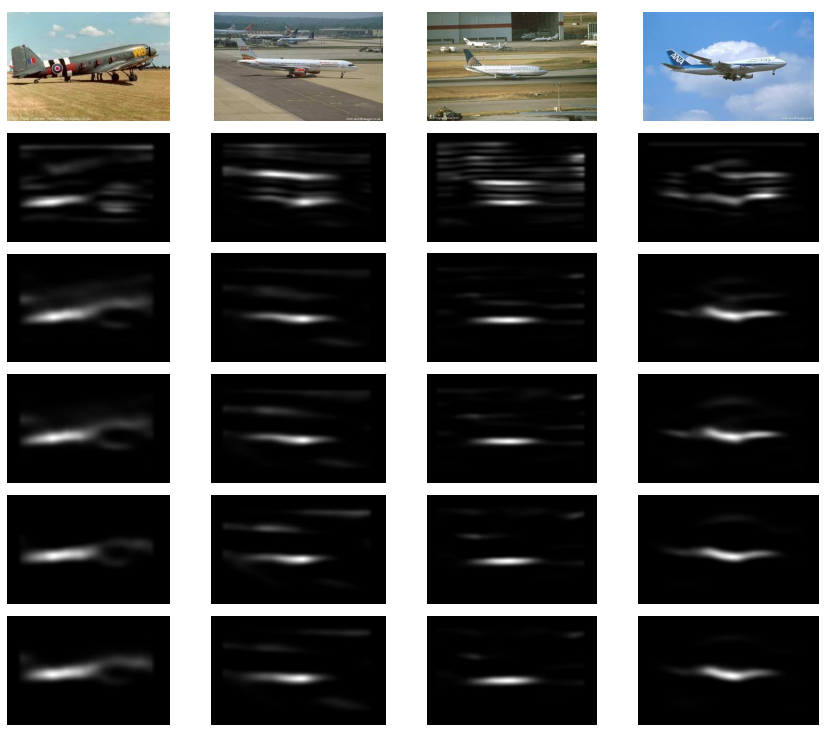

Figure 11. Saliency maps generated with features of different complexity, for the airplane class. From the second to the last row: $k \in\{1,4,8,16,32\}$.

[3] S. Ullman, M. Vidal-Naquet, and E. Sali, "Visual features of intermediate complexity and their use in classification," Nature Neuroscience, Vol.5, No.7, pp. 1-6, 2002.

[4] E. Borenstein and S. Ullman "Learn to Segment," In Proc. ECCV, pp. 315-328, 2004

[5] R. Fergus, P. Perona and A. Zisserman, "Object Class Recognition by Unsupervised Scale-Invariant Learning," In Proc. CVPR, Vol. 2, pp. 264-271, 2003.

[6] C. Harris and M. Stephens, "A combined corner and edge detector," Alvey Vision Conference, 1988.

[7] D.G. Lowe, "Object recognition from local scale-invariance features,” Proc. ICCV, pp. 1150-1157, 1999.

[8] T. Kadir and M.1 Brady, "Scale, Saliency and Image Description," International Journal of Computer Vision, Vol.45, No.2, pp.83-105, 2001

[9] Gy. Dorko and C. Schmid "Selection of Scale-Invariant Parts for Object Class Recognition", Proc. ICCV, pp.634-640, 2003

[10] A. Opelt, M. Fussenegger, A. Pinz and P. Auer, "Weak Hypotheses and Boosting for Generic Object Detection and Recognition", Proc. ECCV, pp. 71-84, 2004

[11] D. Gao and N. Vasconcelos, "Discriminant Saliency for Visual Recognition from Cluttered Scenes," Proceedings of Neural Information Processing Systems (NIPS), 2004 (a)

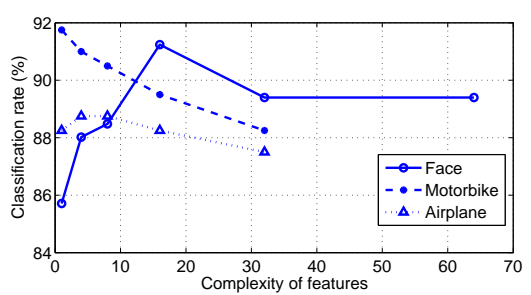

(b)

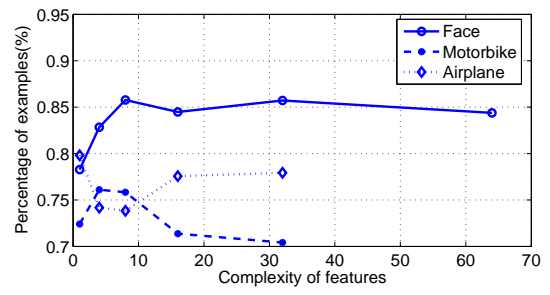

Figure 12. (a) ROC equal error rates for SVM classifer designed at each stage. (b) the percentage of examples accepted after cross-validation step.

[12] N. Vasconcelos, "Feature Selection by Maximum Marginal Diversity," Proceedings of Neural Information Processing Systems (NIPS), 2002

[13] J. Malik and P. Perona, "Preattentive texture discrimination with early vision mechanisms," J Opt Soc Am A, Vol. 7(5), pp. 923-932, 1990

[14] P. Viola and M. Jones, "Robust real-time object detection," Second International Workshop on Statistical and Computational Theories of Vision Modeling, Learning, Computing and Sampling, July 2001.

[15] V. N. Vapnik, The Nature of Statistical Learning Theory, Springer-Verlag, NY, 1995. 